\title{
Signaling pathway switch in breast cancer
}

\author{
Arnaud Guille, Max Chaffanet and Daniel Birnbaum*
}

\begin{abstract}
Next generation sequencing studies have drawn the general landscape of breast cancers and identified hundreds of new, actual therapeutic targets. Two major signaling pathways seem to be altered in a vast proportion of breast cancers. The PI3 kinase/AKT pathway is activated and the JUN/MAPK pathway is repressed. Via the regulation of the cell cycle this metabolic switch impacts on the balance between self-renewal, proliferation and differentiation of the tumor-initiating cells
\end{abstract}

Keywords: Next generation sequencing, Breast cancer, Signaling pathways, Cell cycle, Kinases

\section{Background}

Recent results from next generation sequencing (NGS) studies have established the repertoire of driver gene mutations and copy number alterations (CNA) in breast cancer [1-4]. Nearly 900 cancers representative of all major expression subtypes (basal, luminal A and B, ERBB2 and normal-like) have been studied. Many recurrent mutations have been uncovered. Mutations in TP53, PIK3CA, GATA3 and PTEN genes are among the most frequent. These studies have forever changed our understanding of mammary oncogenesis.

\section{Hypothesis}

Many studies will extend these pioneering ones but it is already possible to speculate further on the NGS data. Data analysis revealed that some alterations (CNA and/ or mutations) never occur in the same tumor, i.e. are mutually exclusive [1-4]. Two main signaling pathways seem to be targeted, the PI3K/AKT pathway and the JUN/ MAPK pathway [1-4]. Alterations in components of the PI3K/AKT pathway (PIK3CA, PIK3R1, AKTs, PTEN, INPP4B...) are mutually exclusive but strikingly, amplification and upregulation of genes encoding receptor-type tyrosine kinases (RTKs) (IGF1R, EGFR, ERBB2) are also (globally) mutually exclusive with alterations of the PI3K/ AKT pathway. This suggests that the primary role of RTK amplification or mutation is to activate the PI3K/AKT pathway. Thus, in the normal mammary epithelium these

\footnotetext{
* Correspondence: daniel.birnbaum@inserm.fr

Centre de Recherche en Cancérologie de Marseille, Oncologie Moléculaire, "Equipe labellisée Ligue Contre le Cancer", UMR1068 Inserm, CNRS UMR7258, Institut Paoli-Calmettes, Aix-Marseille Université, 27 bd. Leï Roure, BP 30059, Marseille 13273, France
}

RTKs are repressed or expressed at a low level and their signaling is primarily oriented toward the JUN/MAPK pathway, whereas when upregulated in tumor cells they stimulate the PI3K/AKT pathway. To obtain this dosage effect could be the reason for the amplification of ERBB2 and FGFR1 genes, although there could be other reasons [5]. It is known that the PI3K/AKT pathway is activated in tumors with mutated EGFR or overexpression of ERBB2 and determines the response to ERBB targeted inhibitors [6]. Within the JUN/MAPK pathway alterations of the components are also mutually exclusive [1]. Components of the JUN/MAPK pathway are inactivated by deletions and mutations, such as MAP2K4 and MAP3K1, or by amplifications, such as $P A K 1$. Most importantly, alterations leading to the activation of the PI3K/AKT pathway and those leading to the inactivation of the JUN/MAPK pathway are mutually exclusive [1]. Finally, not only mutations and genomic rearrangements affect genes encoding components of the two pathways but opposite modifications in expression patterns of these genes could also participate to their switch in breast cancer.

Our hypothesis is that one important consequence of mutations, CNA and modifications of expression is to shift cell signaling in the targeted mammary epithelial cell from an active "JUN/MAPK pathway - inactive PI3K/AKT pathway" state to an active "PI3K/AKT pathway - inactive JUN/MAPK pathway" state.

\section{Testing the hypothesis}

In the diagram shown in Figure 1 we have represented 19 KEGG metabolic and signaling pathways and cell processes altered by mutations in their components in 
the 602 breast cancer samples of the NGS studies for which the molecular subtype had been assigned [1-4]. All pathways and processes are altered, although to different extents. Many potential therapeutical targets can be found in these pathways. Two main signaling pathways are targeted (Figure 2), the JUN/MAPK pathway and the PI3K/AKT pathway [1-4]. Mutations in PTEN, amplifications and mutations in PIK3CA and $A K T$ genes, the MAGI3-AKT3 gene fusion [3], activate the PI3K/AKT pathway whereas mutations in MAP3K1, MAP3K13 and MAP2K4 inactivate the JUN/MAPK pathway. The PI3K/AKT and JUN/MAPK pathways are intimately related and intricate. For example, AKT activation inhibits MAP2K4. This interaction and the mirror effect of the alterations on the two signaling pathways suggest that the PI3K/AKT pathway stimulates the growth of tumor cells whereas the JUN/MAPK pathway has an opposite effect and that the two pathways are the two sides of the same coin.
The downstream effects of the activated PI3K/AKT and inhibited JUN/MAPK pathways are multiple, but at least two could be of primary importance for the behavior of the tumor-initiating cell that fuels the tumor growth. A first major effect could be on the cell cycle. During the G1 phase of the cell cycle, a checkpoint before entering $S$ phase, also called the restriction (R) point, has been defined as an important cell cycle stage controling various cell fates [8] (Figure 3). The G1 phase of the cell cycle has been thus divided into an early, signaling factor-dependent subphase, controled by D cyclins and a late, factor-independent subphase, controled by $\mathrm{E}$ cyclins and a fully inactivated (hyperphosphorylated) RB protein. The JUN/MAPK signaling pathway plays a role in the early G1 subphase, where the cell may be induced into quiescence, senescence or committed to differentiation, depending on the presence of external factors [9]. When entering the late G1 subphase, quiescence, cell death or differentiation are no longer options and the cell 


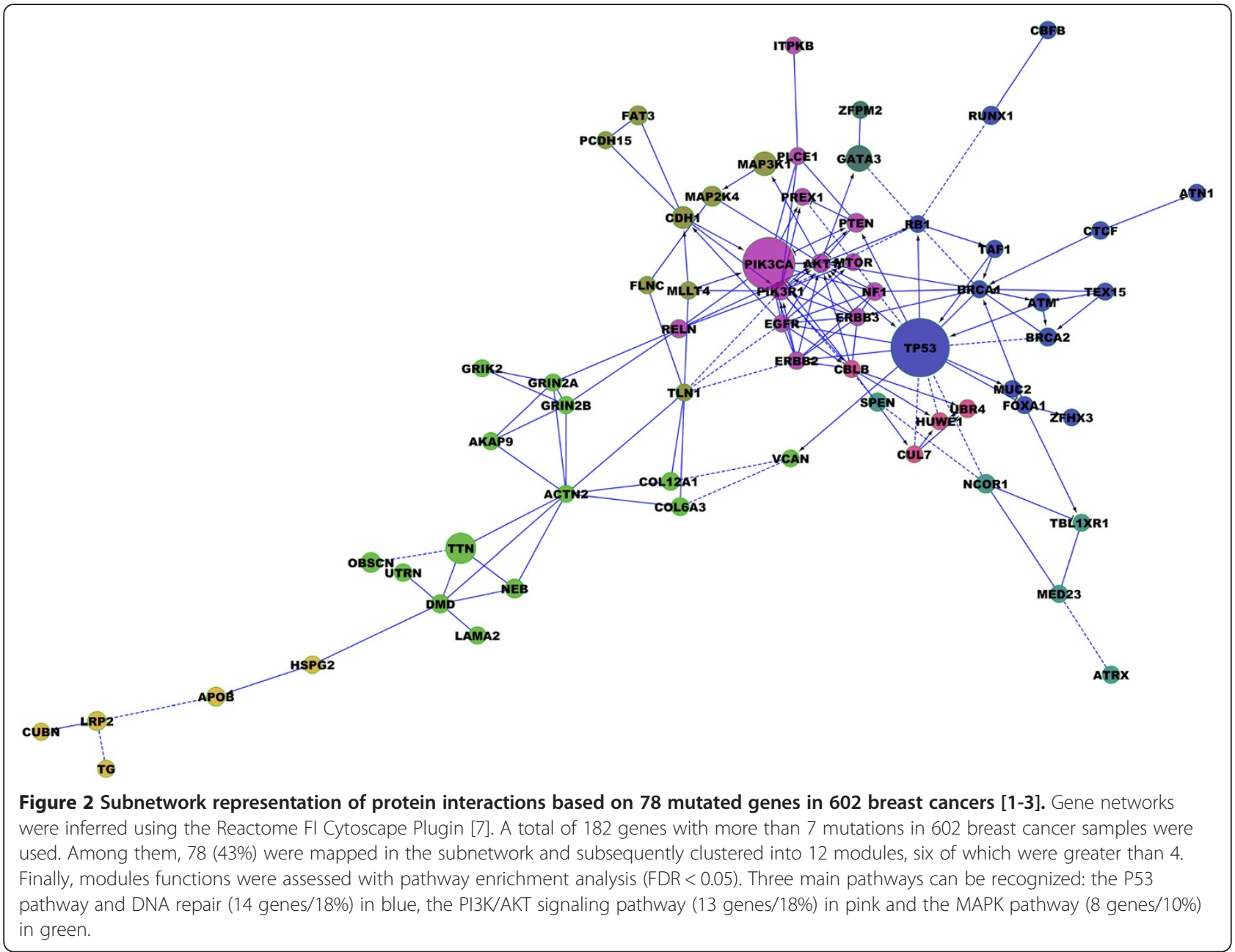

progresses to $\mathrm{S}$ phase and then to either symetric (proliferation) or asymetric (self-renewal) division. At the level of the tumor-initiating cell, the PI3K/AKT pathway could stimulate self-renewal and/or proliferation and the JUN/MAPK pathway cell differentiation or cell cycle arrest. A compromised JUN/MAPK pathway and constitutively activated PI3K/AKT pathway could force the cell to go through the R point and progress to S-phase. In this scheme, amplification of cyclin D1 appears as a particularity; however, it occurs mainly in luminal tumors, which are the most differentiated and less proliferative of all breast tumors. Combined cyclin D1 amplification (or a mutation with similar effect) and PI3K/ AKT pathway would give luminal tumors their particular phenotype. In addition to the signaling pathways, the P53 protein acts as an important checkpoint on the way to the late G1 subphase. Inactivation of P53 (by way of mutations or deletions of the TP53 gene, or another mechanism such as the amplification of the MDM2 gene) is a prominent feature of all subtypes of breast cancer. However, it is more frequent in aggressive breast cancers, especially the basal cases, where alterations of the PI3K/AKT and JUN/MAPK pathways are less predominant. Basal breast cancers display a rearranged genome. P53 inactivation, which has consequences on the stability of the genome beyond those that could result from an activation of signaling pathways, could be the major cause of the basal phenotype.

A second major effect of PI3K/AKT pathway activation and JUN/MAPK inhibition is on the metabolism of the tumor-initiating cells. The latter are particularly resistant to oxidative processes and ROS production. The PI3K/AKT pathway, beside its role on cell growth and survival, regulates a number of metabolic processes including cell glucose uptake and neoglucogenesis in relation with nutrient availability and redox and energy conditions. It plays an important role in the glycolytic phenotype of tumors [10]. Forkhead transcription 


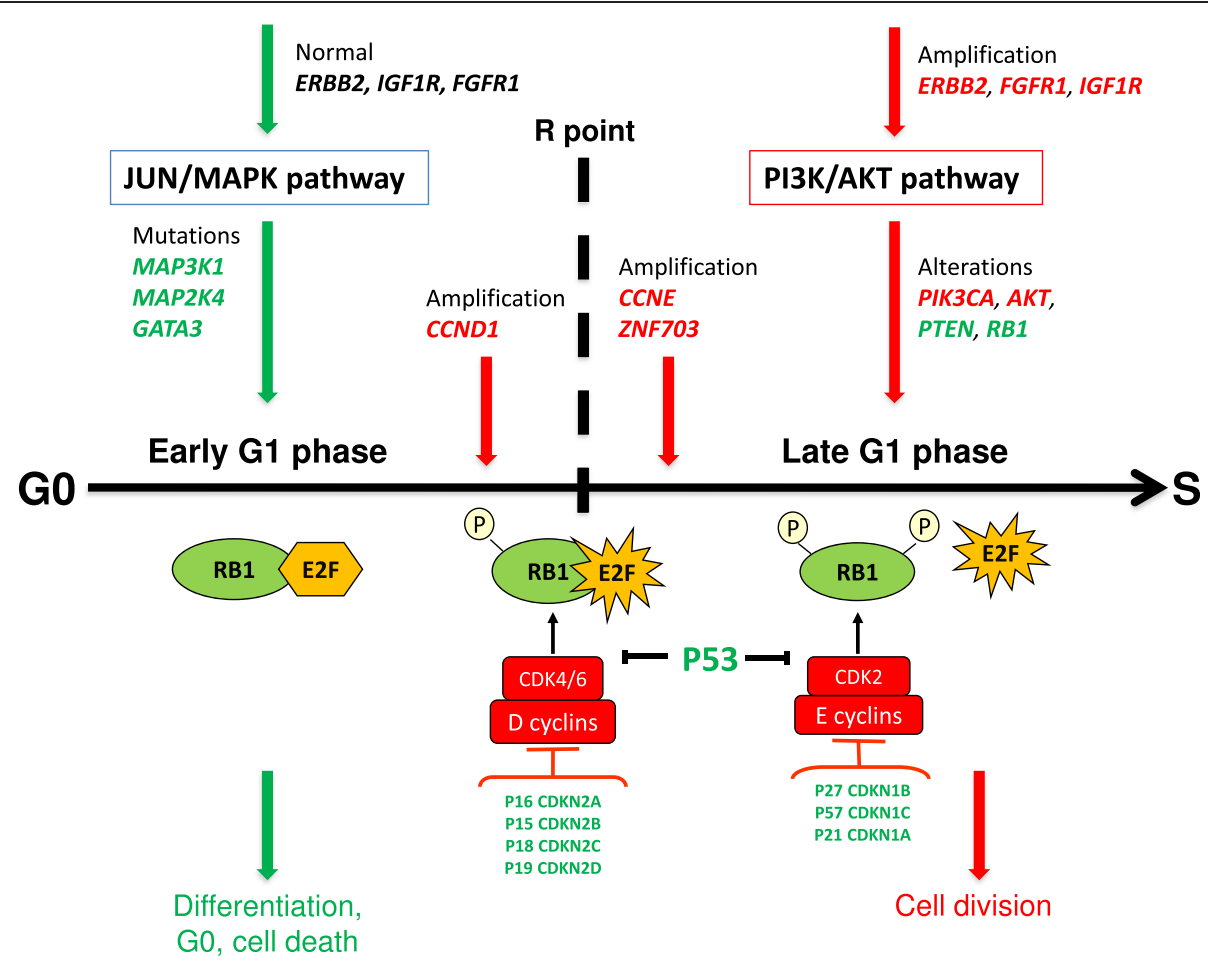

Figure 3 Hypothetical representation of opposite downstream effects of two signaling pathways on the G1 phase of the cell cycle in a breast tumor-initiating cell. During the early G1 subphase the cell integrates signals that may lead it to several fates (differentiation, apoptosis, senescence, quiescence, pause for DNA repair, progression to $S$ phase) [8]. Inhibition of the JUN/MAPK pathway shortens the early G1 subphase rendering the cell independent of differentiation and apoptotic factors. In the same time, activation of the PI3K/AKT pathway induces the cell to progress to late G1 subphase beyond the restriction (R) point; the cell may then undergo symmetric (proliferation) or asymmetric (self-renewal) division. In green: proteins with repressive effect, in red: proteins with activating effect.

factors FOXO are downstream of the PI3K/AKT and JUN/MAPK cascades. FOXOs play a major role in the regulation of both the cell cycle and metabolism [11]. They prevent stem cells to accumulate ROS and allow DNA repair or quiescence. FOXOs are activated by the JUN kinase signaling pathway in the presence of oxidative stress. Conversely, activation of the PI3K/AKT and inhibition of JUN/MAPK pathways overrules the tumor suppressive effect of FOXOs and allows G1 progression. With respect to cell cycle and cell fate decisions FOXO transcription factors could thus represent important integrators of the two signaling pathways that are predominantly altered in breast cancer. They are therefore important targets in mammary oncogenesis. FOXO genes have not been found among the most frequently altered genes in breast cancer in NGS studies [1-4]; they are probably not directly targeted (yet, we have recently found rare mutations and deletions of the FOXO3 gene; Cornen et al., submitted). FOXOs functions may also be modified but maintained to ensure some degree of metabolic regulation in the tumorinitiating cells. FOXOs and P53 act on the same cellular processes and transcription programs, and are intimately cross-regulated [11].

\section{Implications of the hypothesis}

The NGS studies have drawn the general landscape of breast cancers and identified hundreds of new, actual targets. Although the role of many of them needs clarification, especially regarding the transcription factors (e.g. GATA3, RUNX, NCORs...), two major signaling pathways seem to be altered. This is good news since strategies to counter the consequences of pathway alterations are known and could be improved. Inhibiting the PI3K/ AKT pathway appears as a paramount strategy to stop tumor growth [12]. If it fails one could adopt a strategy that takes into account both the PI3K/AKT and JUN/ MAPK pathways but because they are truly interrelated this may not be helpful. Targeting additional alterations or pathways (including synthetic lethal ones) [13] outside the PI3K/AKT and JUN/MAPK pathways could be more successful.

\section{Competing interests}

The authors declare that they have no competing interests.

\section{Authors' contributions}

The hypothesis came to view during discussions between the three authors. All authors read and approved the final manuscript. 


\section{Acknowledgements}

Studies on breast cancer in our laboratory are supported by Inserm, Institut Paoli-Calmettes, Ligue Nationale Contre le Cancer (Label DB) and Institut National du Cancer (ACI2007, AO2008, IVOIRES, and INCa-DGOS-Inserm 6038)

Received: 14 May 2013 Accepted: 20 June 2013

Published: 27 June 2013

\section{References}

1. The Cancer Genome Atlas Network: Comprehensive molecular portraits of human breast tumours. Nature 2012, 490:61-70.

2. Shah P, Roth A, Goya R, Oloumi A, Ha G, Zhao Y, et al: The clonal and mutational evolution spectrum of primary triple-negative breast cancers. Nature 2012, 486:385-399.

3. Banerji S, Cibulskis K, Rangel-Escareno K, Brown KK, Carter SL, Frederick AM, et al: Sequence analysis of mutations and translocations across breast cancer subtypes. Nature 2012, 486:405-409.

4. Ellis MJ, Ding L, Shen D, Luo J, Suman VJ, Wallis JW, et al: Whole-genome analysis informs breast cancer response to aromatase inhibition. Nature 2012, 486:353-360.

5. Birnbaum D, Sircoulomb F, Imbert J: A reason why the ERBB2 gene is amplified and not mutated in breast cancer. Cancer Cell Int 2009, 9:5.

6. Hynes N, MacDonald G: ErbB receptors and signaling pathways in cancer. Curr Op Cell Biol 2009, 21:177-184.

7. Wu G, Feng X, Lincoln Stein L: A human functional protein interaction network and its application to cancer data analysis. Genome Biol 2010, 11:R53.

8. Oxford KW, Scadden DT: Deconstructing stem cell self-renewal: genetic insights into cell-cycle regulation. Nat Rev Genet 2008, 9:115-127.

9. Burdon T, Smith A, Savatier P: Signalling, cell cycle and pluripotency in embryonic stem cells. Trends Cell Biol 2002, 12:432-438.

10. Cairns RA, Harris IS, Mak TW: Regulation of cancer metabolism. Nat Rev Cancer 2011, 11:85-95.

11. Eijkelenboom A, Burgering BMT: FOXOs: signalling integrators for homeostasis maintenance. Nat Rev Mol Cell Biol 2013, 14:83-97.

12. Grunt TW, Mariani GL: Novel approaches for molecular targeted therapy of breast cancer: interfering with PI3K/AKT/mTOR signaling. Curr Cancer Drug Targets 2013, 13:188-204.

13. Eisinger $F$, Sobol $H$, Birnbaum D: Hypothesis: more mutations to cure cancer? Oncol Rep 1999, 6:1189-1190.

\section{Submit your next manuscript to BioMed Central and take full advantage of:}

- Convenient online submission

- Thorough peer review

- No space constraints or color figure charges

- Immediate publication on acceptance

- Inclusion in PubMed, CAS, Scopus and Google Scholar

- Research which is freely available for redistribution 